\title{
Junge Städter zwischen Improvisation und Ausbeutung
}

\author{
Abidjans Handyguthabenverkäufer als Knotenpunkte von Infrastrukturen des \\ digitalisierten Kapitalismus
}

Hannah Schilling

\begin{abstract}
Dieser Artikel nimmt die Handy-Guthabenökonomie in Abidjan in der Côte d'Ivoire zum Ausgangspunkt, um städtische Wertschöpfungsprozesse im digitalen Kapitalismus zu problematisieren. Mithilfe von Konzepten der Infrastruktur und der ökonomischen Praxis zeigt der Artikel, wie soziale Beziehungen, die auf den ersten Blick außerhalb des Arbeitsprozesses stehen, funktional für Wertschöpfungsprozesse werden. Die empirische Grundlage bilden leitfadengestützte Interviews mit rund 30 Männern im Alter zwischen 18 und 35 Jahren, die in der Guthabenökonomie Abidjans arbeiten. Die Analyse arbeitet die Verschränkung von Formen der Zirkulation mit ökonomischen Praktiken heraus. Diese konstituieren (mit) den Guthabenverkauf und damit auch die Kommunikationsinfrastrukturen. Der Artikel verdeutlicht, inwiefern die Befragten ihre Tätigkeit als symbolisches Kapital beim Übergang ins Erwachsenenalter einsetzen. Gleichzeitig nutzen Unternehmen die unbezahlte Arbeit der Guthabenverkäufer für die Erschließung neuer Märkte und zur Wertschöpfung. Im Sinne einer strategischen Kosmopolitisierung der Stadtforschung zeigt der Artikel abschließend Anknüpfungspunkte für die Debatte um digitale Arbeit in der Plattform-Metropolis auf.
\end{abstract}

Ersteinreichung: 4. Mai 2020; Veröffentlichung online: 23. April 2021

An English abstract can be found at the end of the document.

Digitale Technologien verändern die Art und Weise, in der wir konsumieren und produzieren. Technologischer Wandel bringt stets auch neue Formen der Wertschöpfung mit sich. Traditionelle Verwertungsprozesse werden derzeit um Informations- und Kommunikationstechnologien ergänzt, was als „digitalisierter Kapitalismus“ beschrieben wird (Schiller 2014, 1999; Staab 2019; Staab/Nachtwey 2016). Zudem etabliert sich ein neues Produktionsmodell, in dem die Akkumulation gesellschaftlichen Reichtums weniger auf der Produktion von Waren basiert, sondern auf Profiten, die als Rendite aufgrund von Marktbesitz erwirtschaftet werden (Staab 2019: $47 \mathrm{f}$.). Digitale Plattformen wie Uber, Airbnb oder Amazon sind paradigmatische Beispiele für diese „proprietären Märkte“, die laut Philipp Staab den digitalen Kapitalismus kennzeichnen (2019: 43). In diese veränderten Wertschöpfungsprozesse ist auch menschliche Arbeit eingebunden: Die Diskussionen zu Gig Work, Crowd Work und Sharing Economy attestieren eine Auflösung betrieblicher Organisation und Einbindung von Arbeitskraft sowie die Flexibilisierung des Zugriffs auf Arbeitskraft (Kirchner 2019; Schmidt 2016; Staab/Nachtwey 2016; Stefano 2015). Während die Rolle neuer Steuerungsinstrumente für den effizienten Einsatz von Arbeitskraft sowie die 
Disziplinierung aus der Distanz breit erforscht ist - beispielsweise die algorithmusbasierte Aufgabenorganisation, Ratings oder Reputationssysteme (Eyert/Irgmaier/Ulbricht 2020; Griesbach et al. 2019; Wood et al. 2019) -, bleibt die Frage unterbeleuchtet, wie soziale Beziehungen und die dazugehörige Beziehungsarbeit, die auf den ersten Blick außerhalb des Arbeitsprozesses stehen, ebenfalls funktional für Wertschöpfungsprozesse werden (vgl. hierzu jedoch Fourcade/Klutz 2020).

Der Artikel will einen Beitrag zur Beantwortung dieser Frage leisten. Seine Grundlage ist eine Fallstudie zur Prekarisierung junger Erwachsener in der Handyguthabenökonomie in Abidjan (Côte d'Ivoire) zwischen 2015 und 2017 (Schilling 2020).[1] Die Analyse sozialer Dynamiken in dieser informalisierten Erwerbsarbeit soll zeigen, wie Beziehungsarbeit und nicht-marktförmige Austauschbeziehungen in Wertschöpfungsprozesse eingebunden werden.

Städtische Informalität in Kontexten des sogenannten Globalen Südens ist bereits breit erforscht (Hart 1973; Neves/Toit 2012; Roy/AlSayyad 2004; Thieme 2018; Tranber Hansen/Vaa 2004). Ökonomische Praktiken jenseits der formalen Lohnarbeit sind hierbei Teil des analytischen Repertoires. Im Sinne einer strategischen Kosmopolitisierung von Theorien zu Urbanität und städtischen Ökonomien (Robinson 2015, 2002) kann dieses analytische Repertoire auch für die sich im digitalen Kapitalismus verstetigende Auflösung der konzeptionellen Grenzen zwischen Arbeit und Nicht-Arbeit (Richardson 2017) sowie der Vervielfältigung von Arbeitsformen und -orten (Altenried 2017; Ritzer 2015; Rossi 2019) nutzbar gemacht werden.

Empirisch basiert die Fallstudie auf leitfadengestützten Interviews mit rund 30 Männern im Alter zwischen 18 und 35 Jahren, die mehrheitlich in Wohnvierteln des urbanen Mittelschichtsmilieu Abidjans aufwuchsen (namentlich in den Bezirken Yopougon und Koumassi) und dort zum Zeitpunkt der Studie Handyguthaben verkauften. Die Auswahl der Interviewten erfolgte nach einer Kombination aus Schneeballprinzip und theoretischem Sampling. Die Interviews dauerten durchschnittlich 6o Minuten und bestanden aus offenen Fragen zur Organisation des Lebensunterhalts sowie zur Mobilisierung der dazu notwendigen diversen sozialen Beziehungen. Die Interviews wurden ergänzt durch teilnehmende Beobachtungen in der Guthaben-Ökonomie Abidjans sowie durch ExpertInneninterviews mit Akteuren in der Telekommunikationsbranche in der Côte d'Ivoire. Die Interviews wurden transkribiert und auf Grundlage der Grounded Theory ausgewertet (Charmaz 2006; Przyborski/Wohlrab-Sahr 2009). Um die Funktionen des Guthabenverkaufs aufzuzeigen, wurden die für die Organisation des Lebensunterhalts zentralen ökonomischen Praktiken und Beziehungsformen herausgearbeitet.

Im Folgenden führe ich zunächst die Kernkonzepte der Analyse ein und skizziere die Entwicklung des Telekommunikationssektors in der Côte d'Ivoire seit den 1980er Jahren. Anschließend diskutiere ich die empirische Analyse in drei Schritten: Erstens gehe ich auf die Verschränkung von Formen der Zirkulation mit ökonomischen Praktiken ein, die den Guthabenverkauf und damit auch die Kommunikationsinfrastrukturen (mit) konstituieren. Zweitens zeige ich spezifischer, dass ihre Tätigkeit für die Guthabenverkäufer in ihrer Position als junge Menschen im Übergang zum Erwachsenenalter zu symbolischem Kapital wird. Drittens bette ich diese 
Moralökonomien in den Kontext der Unternehmen ein und argumentiere, dass sie auch unbezahlte Arbeit der Guthabenverkäufer legitimieren und den Unternehmen helfen, ihre Produkte zu vermarkten sowie neue urbane Märkte in peripheren NachbarInnenschaften zu erschließen. Abschließend zeige ich Anknüpfungspunkte für die Debatte um digitale Arbeit in der „Plattform-Metropolis“ (Rossi 2019) auf.

\section{Infrastrukturen der Wertschöpfung im digitalisierten Kapitalismus}

Im Folgenden betrachte ich Telekommunikationsnetze als Infrastrukturen des digitalisierten Kapitalismus, oder „Architekturen der Zirkulation“ (Larkin 2013; zitiert in Tonkiss 2015). Diese Infrastrukturen sind nicht identisch mit einem System oder einer Struktur, sondern definiert durch die „Bewegung oder Strukturierung von sozialer Form“ (Berlant 2016: 393). Als Infrastrukturen setzen Telekommunikationsnetze verschiedene Elemente in Beziehung zueinander und machen diese dadurch miteinander verknüpfbar und erreichbar. Dabei bestehen Kommunikationsinfrastrukturen nicht nur aus Funktürmen, Kabelstraßen, Mobilfunk-Endgeräten und Codes. Sie werden vor allem durch menschliche Arbeit aufrechterhalten (Tonkiss 2015). Wie Arjun Appadurai (2014: xiii) feststellt: Infrastruktur umfasst mehr als eine „urbane Technik“, es ist eine Dimension des städtischen Alltags. „[G]ewöhnliche [...] Infrastrukturen der Stadt [bestehen] aus [der] [...] alltäglichen Arbeit der Interaktion, des Austauschs, der Verteilung und der Entsorgung." (Tonkiss 2015: 389, eigene Übersetzung) Das bedeutet, dass Infrastrukturen auch durch menschliche Arbeit vermittelt und untermauert werden. Diese produziert und verteilt materielle Güter, zirkuliert und kanalisiert Informationen, sammelt Treibstoff und Wasser, erzeugt Energie, erbringt Transportleistungen oder entsorgt den anfallenden Abfall (ebd.).

Dieser analytische Zugang aus der urbanen Infrastrukturforschung lenkt den Blick auch auf Arbeit jenseits formaler Lohnarbeit, die mit dem Begriff der ökonomischen Praktiken (Bourdieu 2000: 23; Smith/Stenning 2006; Gibson-Graham 2008) greif- und sichtbar gemacht werden kann. Im Allgemeinen sind Praktiken „routinierte Arten und Weisen, in denen Körper bewegt, Objekte gehandhabt, Subjekte behandelt, Dinge beschrieben und die Welt verstanden werden“ (Reckwitz 2002: 249 f., Übersetzung der Autorin).[2] Ökonomische Praktiken umfassen all jene Praktiken, die die „Befriedigung menschlicher Bedürfnisse in einem bestimmten Kontext" (Hann 2018: 2) organisieren. Dies reicht vom Kaufen und Verschenken von Ressourcen bis zur Einforderung von Ressourcen auf der Basis institutionalisierter Anrechte, etwa als BürgerIn, Teil einer Gemeinschaft etc. (Zelizer 1996). Tonkiss beschreibt, dass Infrastrukturen aus solch einer Kreuzung und Verknüpfung diverser Ökonomien bestehen: „Infrastrukturen, indem sie verschiedene Dinge - Materialien, Ressourcen, Kapital, Informationen, Menschen - in Umlauf bringen, sie ,verknüpfbar' machen, umfassen verschiedene Ökonomien von Investitionen, Eigentum, Austausch und Nutzung." (Tonkiss 2015: 384) Dabei materialisieren sich Infrastrukturen nicht nur in Form von Elektrizitätsnetzwerken, Wasserleitungen oder Straßenkreuzungen. Auch Aktivitäten von StadtbewohnerInnen können als soziale Infrastrukturen gefasst werden, 
an denen sich verschiedene Sozialitäten und Räume ökonomischer und kultureller Operationen kreuzen und dadurch Kollaborationen an den Rändern städtischen Lebens ermöglichen (Simone 2004).

Ausgehend von der bestehenden Infrastrukturforschung, die vor allem danach fragt, wie Praktiken verschiedener Akteure und Agenten Infrastrukturen konstituieren und wie städtische Versorgung organisiert wird (Bennett 2005; Star 1999; Graham 2009; Graham/Marvin 2002; Angelo/Hentschel 2015; Plantin et al. 2018: 269), sollen im Folgenden Wertschöpfungsprozesse sichtbar gemacht werden. Mit dem Fokus auf Infrastrukturen rücken Momente des Verschränkens und Verknüpfens von Elementen in den Vordergrund. Das bietet die Chance, Prozesse außerhalb offizieller bezahlter Arbeit in die Analyse der Wertschöpfung miteinzubeziehen. Dazu müssen die Praktiken und Dinge nicht die Form eines Warenaustauschs annehmen. Spannend ist, zu untersuchen, wie diese - ganz im Sinne einer Infrastrukturperspektive übersetzt und kommensurabel gemacht werden (Tsing 2013). Verónica Gago und Sandro Mezzadra beschreiben „neue extraktive Operationen“ (Gago/ Mezzadra 2017), die sich von den Prinzipien der Ausbeutung von Lohnarbeit unterscheiden, da hier Kapital in die marginalisierten Lebensbereiche und sozialen Infrastrukturen marginalisierter Bevölkerungen eindringt, um Wert zu entwenden. Die Abschöpfung von Wert geht über Situationen hinaus, in denen Individuen explizit erleben, dass sie mit ihrer Arbeitskraft und Identität als ArbeiterInnnen in kapitalistische Wertschöpfung eingebunden sind (Mezzadra/Neilson/Cukier 2018: 108). Dadurch entsteht eine Diskrepanz zwischen den extraktiven Operationen des Kapitals und den subjektiven Erfahrungen von Ausbeutung. Zusätzlich sind die „neuen extraktiven Operationen" davon gekennzeichnet, dass ArbeiterInnen nicht durch Arbeitsverträge an vom Kapital organisierte Räume gebunden sind (etwa Fabriken), sondern, im Gegenteil, kapitalistische Akteure die soziale Kooperation, die sie ausbeuten, nicht mehr selbst organisieren (Gago/Mezzadra 2017: 579). Dies rückt Kooperationsformen jenseits formalisierter Arbeitsverhältnisse sowie die ihnen zugrunde liegenden Dynamiken in den Vordergrund.

Informalisierte Kooperation und Arbeit wurden lange Zeit entweder außerhalb von Marktbeziehungen und kapitalistischer Wertschöpfung verortet (vgl. kritisch dazu Lourenço-Lindell 2001) oder als exemplarisch für Unternehmertum und Marktgesetze hervorgehoben (zu dieser Kritik vgl. Marcelli/Williams/Joassart 2010: 10). Im Folgenden soll jedoch vielmehr gezeigt werden, dass Sphären sozialer Kooperation in die Wertschöpfung eingebunden werden, in denen Transaktionen weder marktförmig noch formalisiert sind (vgl. auch Rekhviashvili 2015). Die Black Box Informalität zu öffnen, bedeutet hier, ökonomische Praktiken und Beziehungsformen, speziell informalisierter Arbeitsbeziehungen, zu differenzieren und genauer zu beschreiben (Lourenço-Lindell 2001; Viti 2013): Ökonomische Praxis kann die pragmatische Suche nach neuen Gelegenheiten (Jeffrey/Dyson 2013: R1) umfassen. Das kann auch bedeuten, dass Akteure sich durch möglichst viele Transaktionen versuchen ins Spiel zu bringen, ohne sich allzu stark festzulegen (Simone 2004). In Handelsbeziehungen können die Fähigkeit der KundInnenenbindung sowie Formen der Sozialität jenseits des homo oeconomicus [3] wertvoll werden. Schließlich rücken nicht-staatliche Formen von Autorität in den Blick, in denen Beziehungen zwischen 
Angehörigen verschiedener Generationen Hierarchien sowie die Verteilung von Ressourcen und Gelegenheiten strukturieren (Cutolo 2015; Neves/Toit 2012; Viti 2013). Derlei ökonomische Praktiken verweben sich zu städtischen Infrastrukturen und charakterisieren das, was Simone als „Stadt im Entstehen" (wörtlich: in the making) bezeichnet (Simone 2010: 3). Wie diese Praktiken in kapitalistische Wertschöpfung mit eingebunden werden, durch sie aber zugleich Räume der Wiederaneignung und Improvisation entstehen (Rossi 2019), soll im Folgenden untersucht werden.

\section{Kleine Geschichte der mobilen Kommunikationsnetzwerke in Abidjan}

Mobiltelefone sind aus dem städtischen Alltag Abidjans heutzutage nicht mehr wegzudenken. Eine Mehrheit der StadtbewohnerInnen nutzt sie; in wenigen Jahrzehnten etablierte sich die Telekommunikation als wichtiger Wirtschaftssektor und das Mobiltelefon verwandelte sich in ein städtisches Accessoire mit hohem symbolischem Kapital (Matlon 2014; Kamga 2012; Hamanys Broux de Ismael 2016; Kiyindou/Anaté/Capo Chichi 2015). Die Bereitstellung von Kommunikationsdiensten hat sich seit den 1990er Jahren stark verändert: Im Rahmen der Strukturanpassungsprogramme in der Côte d'Ivoire wurde 1997 die Festnetztelefonie privatisiert und der Markt für externe Mobilfunkanbieter geöffnet (Laffont/N'Guessan 2002: 6 f.). Diese Entwicklung veranschaulicht die Abkehr vom Ideal einer öffentlichen Versorgung, das den Zugang zu Kommunikationsinfrastrukturen in staatliche Hand legte, hin zu einem splintering, d. h. einer Aufspaltung in verschiedene, private und konkurrierende Mobilfunknetze (Graham/Marvin 2002; Plantin et al. 2018: 300 f.). Die Investition in den Ausbau dieser Netze und die Bereitstellung von Kommunikationsinfrastruktur wird so vor allem zu einer Frage von Angebot und Nachfrage. 2015, zu Beginn der Fallstudie, gab es in der Côte d'Ivoire fünf Unternehmen, die der Bevölkerung Mobilfunkdienste anboten: Orange, MTN, Moov, Green und Comium, wobei Orange gemessen an den Verkaufszahlen einen Marktanteil von fast 50 Prozent hielt (ARTCI 2015: 22).

Heute lässt sich eine neue Phase der Telekommunikationsunternehmen beobachten: Zwar sind sie immer noch Anbieter von Kommunikationsdienstleistungen, stellen Tarife und Technologien zur Verfügung. Darüber hinaus sind sie jedoch zu einer Koordinationsstelle und zu Vermittlern in einem Geflecht von Dienstleistungen geworden. An erster Stelle sind hier die Etablierung mobiler Geldtransfer-Dienste und die Entwicklung eigener virtueller Währungen zu nennen (z. B. „Orange Money“), die als Guthaben auf SIM-Karten eines Mobiltelefons geladen werden. Mit diesem Guthaben können Rechnungen beglichen, Geld angelegt sowie Kredite aufgenommen werden. Institutionalisiert wurden diese Finanzdienstleistungen durch die Gründung der virtuellen Orange Bank.[4] Die Unternehmensgruppe Orange investiert in der Region Westafrika in Start-ups mit dem OrangeFabLab. ci[5] und kooperiert als Zahlungsplattform mit Versicherungen[6] und Online-Lieferdiensten.[7] Telekommunikationsunternehmen wie Orange können damit zunehmend auch als digitale Plattformen betrachtet werden: Sie verknüpfen ZahlungsdienstleisterInnen und andere digitale DienstleisterInnen mit KundInnen. Diese nutzen die Infrastruktur für ihre 
Transaktionen (Kendall et al. 2012). Viel deutet darauf hin, dass hier „soziotechnische Ökosysteme“ (Dolata 2015: 511) entstehen, die ihre Produkte und Serviceleistungen ausbauen: Von TV-Abonnements über Smart-HomeTechnologie bis hin zu Kreditprodukten und Krankenversicherungen kann alles Mögliche über die digitale Plattform und die virtuelle Währung des Handyguthabens bezahlt und verwaltet werden. Durch hohe Transaktionskosten im Falle eines Anbieterwechsels werden KundInnen an ein Unternehmen gebunden.

Die Praktiken von Handyguthaben-VerkäuferInnen sind wichtige Akteure bei der Etablierung von Infrastrukturen des digitalisierten Kapitalismus. Ihre Praktiken sollen im Folgenden im Vordergrund stehen. Um in meinem Untersuchungszeitraum (2015-2017) in Abidjan Handyguthaben zu verkaufen, brauchte es nicht viel. Eine SIM-Karte, ein Mobiltelefon und ein Netzwerk an potenziellen KäuferInnen reichte aus, um Gesprächsguthaben zu verteilen und damit Gewinn zu machen. Für den Verkauf von 10.00o CFA-Franc Guthaben erhielten die VerkäuferInnen vier bis sechs Prozent Provision in Form von Guthaben zurück auf die SIM-Karte, von der die Transaktion ausgelöst wurde. Guthaben verkaufen konnte so prinzipiell jedeR - dazu war weder eine feste Ladenstruktur noch eine Ausbildung erforderlich. Für den Erwerb einer SIM-Karte reichte die Identifikation mit einem Personalausweis aus. In der Praxis wurden SIM-Karten allerdings häufig an Dritte weitergegeben, die dann wiederum damit Guthaben verkauften. Es gab ganz unterschiedliche Verkaufsstellen und Konstellationen, in denen Guthaben zirkulierte: Es konnte als zusätzliches Produkt in einem Ladensortiment angeboten oder über bestehende Netzwerke zu Hause verkauft werden. In den 2000er Jahren hat sich dabei eine spezifische Verkaufskonstellation etabliert: die sogenannte cabine (wörtlich: Telefonkabine). Als die Mobiltelefonie in Abidjan geläufig wurde, entwickelten die BewohnerInnen der Stadt eigene Geschäftsideen, um Kommunikationsdienste anzubieten: Manche boten anderen gegen Bezahlung ihre Telefone an, um damit Anrufe zu tätigen oder SMS zu verschicken. Telekommunikationsunternehmen boten Unternehmen rabattierte Tarife an, wenn sie Abonnements für sämtliche MitarbeiterInnen abschlossen. Diese Tarife wurden dann auch von Einzelpersonen genutzt, die damit Guthaben vergünstigt einkaufen und teurer weiterverkaufen konnten. Damit war die ,private‘ Telefonkabine geboren und löste die öffentliche Telefonzelle ab (vgl. Schilling/Dembélé 2019: 124 f.). Auch wenn sie inoffiziell war, wurde sie von den Unternehmen geduldet und später sogar gefördert, wie wir später sehen werden. Unternehmen wie Orange steuerten zunehmend die Verteilung von Guthaben, indem sie mittels Plattformtechnologien Transaktionen an den cabines nachverfolgten, städtische Gebiete in Zonen einteilten und die Verteilung von Guthaben an cabines über firmeneigene Zulieferer formalisierten. Dadurch konnten sie das Guthabengeschäft aktiv gestalten und strategisch ausbauen (Kamga 2012: 378; Nassa Dabié 2012; Touré 2010).

\section{Die Kunst des Guthabenverkaufens: Eine Praxis zwischen Warenzirkulation und Moralökonomien}

Als ,Knotenpunkte einer Kommunikationsinfrastruktur involvieren die cabines ganz unterschiedliche ökonomische Praktiken. Der Zugang zu mobiler 
Kommunikation ist auch in einer privatisierten Netzstruktur eingebettet in ökonomische Praktiken und Märkte mit unterschiedlichen Währungen und Wertigkeiten. Diese Zirkulationen verdeutlichen, welche Ressourcen und Fähigkeiten relevant sind, um sich in informalisierten Erwerbskontexten zu organisieren.

Séverin, 25 Jahre alt, traf ich an einem Straßenrand im Abidjaner Stadtviertel Koumassi. Er saß neben einem Schild, das den Tarif seines Guthaben-Transfers anpries, eine Handvoll Handys lagen auf seinem Schoß. Auch wenn Séverin seine Verkaufsstelle selbstständig betreute und keinen Vorgesetzten hatte, war seine Tätigkeit dennoch in soziale Infrastrukturen eingebettet. Diese waren die Voraussetzung dafür, dass er überhaupt unternehmerisch handeln konnte. Séverin erzählte mir, dass er das Startkapital für seinen Guthabenverkauf von einer Nachbarin erhalten hatte, zu der er eine innige Beziehung als bon pétit (wörtlich: der gute Kleine) etabliert hatte - eine Bezeichnung für Junior (Cutolo 2015). Das Prinzip der Seniorität legitimiert asymmetrische Beziehungen, in denen Macht, Prestige sowie die Kontrolle über Ressourcen jenen zuteilwerden, die mit Senioritätsbezeichnungen addressiert werden. Séverin schilderte dies folgendermaßen: „Sie wurde wie eine Mutter und eine große Schwester für mich." Die Beziehung fundierte auf Séverins Respekt und Vertrauen gegenüber der Nachbarin: „Sie testete mich, zum Beispiel gab sie mir einen Umschlag mit Geld, und sagte: ,Junior, pass darauf auf.' Und dieser Umschlag blieb dann ein paar Monate bei mir, und wenn sie danach fragte, gab ich ihr den Umschlag mit dem Geld - und so begann sie mir zu vertrauen." (Interview mit Séverin, Koumassi, 3. März 2016). Aus der gelebten Zuverlässigkeit wuchs Vertrauen zwischen Séverin und seiner Nachbarin im Rahmen einer Junior-Senior-Beziehung (Eisenstadt/Roniger 1980), also einer Beziehung, die auf asymmetrischer Reziprozität beruht. Die Nachbarin unterstützte Séverin mit dem Startkapital und der Ermahnung, nicht „faul“ zu Hause zu sitzen. Séverin brachte ihr dafür Gehorsam und kleinere Gefälligkeiten entgegen. Als Mikro-Unternehmer war er in diesem Sinne weniger ein individuell agierender Nutzenmaximierer à la homo oeconomicus, sondern stattdessen in ein Netz von Beziehungen eingebunden.

Auch die Organisation eines Verkaufsplatzes war verbunden mit der Mobilisierung sozialer Beziehungen, die über Markttransaktionen oder entpersonalisierte professionelle Beziehungen hinausgehen. Als der 24-jährige Boris und sein gleichaltriger Kollege in ihrem Wohnviertel einen Ort suchten, an dem sie Guthaben verkaufen können, war es zunächst schwierig, einen Platz am Straßenrand zu ergattern. Sie hatten sich eine Stelle in ihrer NachbarInnenschaft ausgesucht, direkt vor einem Friseursalon, am Rande des lokalen Wochenmarktes. Um mit der Besitzerin des Salons ins Gespräch zu kommen und einen guten Deal zu erreichen, benötigten sie die Zustimmung der Autoritäten der NachbarInnenschaft, der grandes personnes (wörtlich: großen Personen), wie Boris sie nennt. Die beiden Verkäufer bemühten sich, die Salonbesitzerin mit einem „pardon“ (wörtlich: Entschuldigung) davon zu überzeugen, ihnen den Platz zu geben. Das pardon ist eine ritualisierte Kommunikationsform (Fancello/Mary 2018), die in die Erwartung generalisierter Reziprozität eingebettet ist. In Konfliktsituationen, im Ringen um einen Kompromiss, appellieren diejenigen, die um Entschuldigung bitten, an 
die zukünftige Bedürftigkeit ihres Gegenübers. Boris erklärte: „[Die Person] erniedrigt sich Dir gegenüber, und da möchtest Du nicht kämpfen, Dich widersetzen. Morgen kannst Du [der Person] wieder begegnen, irgendwo, und Du wirst bedürftig sein, und wenn Du [sie] um Hilfe fragst, wird [sie] sich daran erinnern, dass Du dickköpfig warst, und Dir nicht helfen." (Interview mit Boris, Yopougon, 8. März 2016). Die beiden Verkäufer praktizierten das Prinzip der generalisierten Reziprozität: Sie baten die Salonbesitzerin um Entschuldigung und formulierten anschließend ihren Wunsch, vor deren Salon Guthaben zu verkaufen. Die Besitzerin willigte ein. Auch die kollegialen Beziehungen der GuthabenverkäuferInnen beruhten auf Konventionen jenseits rechtlich kodifizierter und offiziell anerkannter Regeln: Boris beschrieb die Zusammenarbeit mit seinem Kollegen im „Unternehmen“ der cabine als „brüderlich“ und „freundschaftlich“ sowie von Gegenseitigkeit und Gleichheit geprägt. Wir sehen hier eine Form von Freundschaft, die keine affinitätsbasierte Freizeitgemeinschaft zwischen Individuen ist, sondern in der stattdessen materieller Austausch und Soziabilität unmittelbar miteinander verschränkt sind (Mains 2013).

Der Guthabenverkauf war in diesem Sinne kein Freizeitspaß befreundeter Nachbarsjungen. Den Interviewten ging es darum, aus ihren (knapp) vorhandenen Mitteln monetäre und soziale Ressourcen zu schöpfen. Das erinnert an das hustling (sich durchschlagen bzw. herumtreiben), das Tatiana Thieme (2018) treffend als ökonomische Praxis konzeptionalisiert, mit der junge Erwachsene versuchen, auf pragmatische Art und Weise Gelegenheiten beim Schopfe zu greifen, über die Runden zu kommen, sich zu realisieren - und zwar jenseits formeller Einkommensquellen sowie formaler Bildungs- und Karrierewege. Séverin beispielsweise lachte, als ich ihn fragte, wie lange er das Handy schon hatte, mit dem er Guthaben verkaufte: „Dieses Telefon? So an die drei Tage [...] ich verkaufe alles!“ (Interview mit Séverin, Koumassi, 3. März 2016). Zuvor hatte er ein Handy der Marke Samsung besessen, das er verkauft hatte, um eine „andere Aktion voranzutreiben“ (französisch: gérer un mouvement), wie er es ausdrückte (ebd.). Und um nicht allzu große finanzielle Engpässe zu haben, hatte Séverin sich dann jenes gekauft, das er während des Interviews in seinen Händen hielt. Dies stünde aber auch zu Verkauf, falls ich interessiert sei, so Séverin auf meine Nachfrage. Die Handys, mit denen Séverin Guthaben an seine KundInnen verschickte, waren also dreierlei: erstens Arbeitsmittel zum Guthabenverkauf, zweitens ein alltäglicher Gebrauchsgegenstand und drittens verkäufliche Waren. Die Handys speicherten somit Wert und wurden zu einer Art Kapital, das wieder flüssig gemacht werden konnte. Mit diesem Geld konnten dann wieder andere Geschäfte - oder mouvements (wörtlich: Bewegungen), wie es bei Séverin und anderen jungen BewohnerInnen Abidjans heißt - getätigt werden. Diese Zirkulation von Waren und Geld war für PassantInnen und BeobachterInnen wie mich nicht offensichtlich - es gab keinerlei Preisschilder oder Ausstellung von Objekten, mit denen üblicherweise Märkte sicht- und greifbar werden (Muniesa/Millo/Callon 2007: 2). Stattdessen bedurfte es des entsprechenden Know-hows zur Einschätzung der Besitzverhältnisse, innerhalb derer die Objekte zirkulierten. Welcher Preis für ein Handy der ,richtige‘ ist, war auch abhängig davon, wie die jeweils andere Person (VerkäuferIn oder KäuferIn) gelesen wurde und in welcher Beziehung beide Personen zueinander standen. 
Dem bereits erwähnten Boris wurden beispielsweise in seiner cabine des Öfteren Handys zum Kauf angeboten. PassantInnen kamen auf ihn zu und wollten ihre Telefone verkaufen, um an Bargeld zu kommen. Boris beschrieb, dass er bei solchen Angeboten aufpassen müsste, den Preis richtig einzuschätzen, um nicht aus Versehen in einen Eigentumskonflikt zu geraten: Denn falls die Telefone zu günstig angeboten würden, wäre das ein Indiz dafür, dass sie gestohlen worden waren. In diesem Fall hätte er später für den Diebstahl zur Rechenschaft gezogen werden können. Wegen dieser schwierigen Einschätzung der Besitzverhältnisse erzählte Boris, nur Handys von Personen zu kaufen, die er kenne: „[H]ier [...] wissen wir, wem wir die Telefone bezahlen, jemand mit einem [moralischen] Bewusstsein, jemand, den wir in der NachbarInnenschaft kennen, sodass wir keine Probleme mit der Polizei bekommen." (Interview mit Boris, Yopougon, 8. März 2016)

Die Zirkulation von Objekten beruht also auch auf moralischen Ökonomien, in denen ein gutes ,Bewusstsein' ebenso wichtig ist wie eine Einschätzung des ,richtigen' Preises. Märkte sind Netzwerke des Waren-Austauschs (Slater/Tonkiss 2001: 38), eine Art von agencements (ökonomischen Konstellationen), die durch Zirkulation, Preisgestaltung und Austausch charakterisiert sind. Solche agencements lösen „Dinge [voneinander] [...] und verbinden sie mit anderen Dingen. Dasselbe geschieht (physisch oder moralisch), mit ihren gegenseitigen Pflichten und mit ihren Beziehungen zu den Dingen“ (Muniesa/Millo/Callon 2007: 4, eigene Übersetzung). Die Marktkonstellationen, der die Kommunikationsinfrastruktur zugrunde liegt, machen deutlich, dass der Besitzstatus von Objekten oftmals ambivalent und unstet ist. Ihr Austausch wird durch das implizite Wissen über die monetäre und moralische Wertigkeit von Dingen, aber auch von Personen reguliert.

Darüber hinaus zirkulieren Guthaben und Mobiltelefone im Alltag der befragten GuthabenverkäuferInnen auch als Geschenke, als Gefälligkeiten, die in einer Ökonomie der Gegenseitigkeit (Gudeman 2008) verankert sind. François, ein weiterer Verkäufer, verstetigte wie viele andere meiner Gesprächspartner Freundschaftsbeziehungen durch die Transaktion von Guthaben. „Um die Freundschaft zu erhalten, gebe ich ihnen kostenlos Guthaben - sie können das dann später bezahlen. Dadurch bleibt eine Freundschaft zwischen uns, ein Vertrauen“, erklärte François (vgl. Interview mit François, Yopougon, 19. Februar 2016). Die Transaktion von Ressourcen als Geschenk sichert das Bestehen einer Beziehung, indem es Schulden schafft (vgl. Viti 2013: 62 ff.): „Es besteht eine Beziehung zwischen einem Spender, der sich [...] in einer Gläubigerposition befindet, und einem Empfänger, der sich [...] in einer nachrangigen Schuldnerposition befindet, und verpflichtet ist, die so eingegangene Schuld früher oder später zu begleichen.“ (Marie 1997: 210, eigene Übersetzung) Schuldbeziehungen verbinden die Gegenwart mit der Zukunft (vgl. Guérin 2014: 41) und sind ein strukturierendes Prinzip von Reziprozität.

Diese Beobachtungen zeigen die vielfältigen ökonomischen Praktiken, die an die offiziellen Kommunikationsnetze andocken. Die Kunst des Guthabenverkaufs ist weit mehr als die bloße Übergabe von Kommunikationsguthaben gegen eine feste Geldsumme. Auch Gabenökonomien, moralische Bewertungen und Beziehungen zwischen Personen spielen bei den Transaktionen eine Rolle. Konventionen, wie beispielsweise das Prinzip der 
Brüderlichkeit oder das pardon, nicht-staatliche Autoritätsbeziehungen wie die Junior-Senior-Beziehung oder nachbarInnenschaftliche Ältestenräte strukturieren die Möglichkeiten des Zugangs zu Ressourcen, die Arbeit als GuthabenverkäuferIn sowie die Verteilung von Guthaben. Im Zusammenhang mit der Diskussion um digitale Technologien im urbanen Raum verweist dies auf die Vielfalt ökonomischer Praxis, die untersucht werden muss: Kommunikationsinfrastrukturen werden in sozialen Praktiken aufrechterhalten, und obwohl es privatwirtschaftlich organisierte Netze sind, gibt es gerade in Momenten des Guthabenverkaufs selbst auch Transaktionslogiken, die den Zugang zu Guthaben nicht auf der Grundlage von Preisbildungen regulieren.

\section{Für den guten Ruf: Guthabenverkauf als Statusarbeit der jungen städtischen Mittelschicht}

Die Guthabenverkäufer, die im Zentrum meiner Analyse stehen, sind mehrheitlich junge Männer, die in Vierteln der urbanen Mittelschicht Abidjans groß geworden sind.[8] Mit ihrem Alter zwischen 18 und 35 Jahren repräsentieren sie eine Bevölkerungsgruppe in der Côte d'Ivoire, die besonders stark von Arbeitslosigkeit betroffen ist (vgl. u. a. Kouakou/Koba 2015). Statistische Kategorien können auch andeuten, wie Arbeit symbolisch mit Status verknüpft wird. In der kolonialen Côte d'Ivoire erklärte die Verwaltung formale Lohnarbeit zur einzigen offiziellen Kategorie von Arbeit und grenzte sie undifferenziert von informellen Tätigkeiten ab (Bazin/Gnabéli 1997). Die Kolonialverwaltung, und später auch der postkoloniale unabhängige Staat, waren die Hauptarbeitgeber, was der Côte d'Ivoire auch das Label einer „Beamtenrepublik“ (Matlon 2016) verschaffte. Als PolizistIn, SekretärIn, KrankenpflegerIn oder LehrerIn für den Staat zu arbeiten, bedeutet somit nicht nur eine finanzielle Absicherung für den oder die EinzelneN, sondern vor allem Zugang zu umfassenden Statusrechten der urbanen Mittelschicht (Manou-Savina et al. 1985; Matlon 2016; Mbembe/Roitman 1995). Die Jugendarbeitslosigkeit ist damit auch eine Krise der urbanen Mittelschicht: Vor allem gut ausgebildete junge Erwachsene finden nur schwer eine erste Beschäftigung auf dem formalen Arbeitsmarkt (Kouakou 2011; Kouakou/ Koba 2015). In dieser Situation wird der Guthabenverkauf zu einer temporären Aktivität, um über die Runden zu kommen.

Die Mehrheit der Interviewten betonte, dass sie den Guthabenverkauf nicht als Arbeit betrachte, sondern als kurzfristigen Geldverdienst, bis sie Arbeit finden, eine Aufnahmeprüfung für den Staatsdienst bestehen oder ihr Studium fortsetzen können. Die Befragten bauten sich mit dem Verkauf keine langfristige professionelle Identität auf (Schilling 2020). Dennoch verhandelten die jungen Männer mit ihrer Tätigkeit auch ihre „soziale Existenz" (Ferguson/Li 2018), also ihre Anerkennung als Mitglieder von NachbarInnenschaften und Verwandtschaften. David, ein weiterer Verkäufer, erklärte dazu:

„Nun, [mit der cabine anzufangen] hatte einen positiven Einfluss. [...] Sie [die NachbarInnen] wissen, dass Du nicht stiehlst. Das ist ein Punkt. Und sie wissen, dass Du geschäftig und bemüht bist [im Original: tu te débrouilles], denn die Tatsache, dass Du Dich hinsetzt, 
Guthaben verkaufst, bedeutet, dass Du mutig bist. Denn um das zu tun, muss man Geduld haben. Das ist auch ein gutes Zeugnis [im Original temoignage] [für Deinen guten Ruf].“ (Interview mit David, Koumassi, 13. Oktober 2017)

In Davids Selbstbeschreibung wird die Guthabenverkaufsstelle zu einem Ort, an dem er seine Reputation als junger, unterbeschäftigter Mann zu reparieren vermag. Für David (und andere Interviewte) ist es eine Möglichkeit, in der NachbarInnenschaft als ein junger Stadtbewohner wahrgenommen zu werden, der nicht deviant ist, sondern den Erwartungen an junge Männer entspricht: strebsam, ausdauernd und ehrlich.

Die Guthabenverkaufsstellen befinden sich möglichst gut sichtbar und zugänglich an häufig frequentierten Plätzen. Zudem ist das Guthaben für die StadtbewohnerInnen generell eine grundlegende Ressource, da es Zugang zu Kommunikation ermöglicht. Diese beiden konstitutiven Merkmale der Guthabenverkaufsstellen begünstigen, dass die jungen Erwachsenen sich als „öffentliche Charaktere“ etablieren und in Szene setzen können. Dies beschreibt in Anlehnung an Jane Jacobs eine Person, die „häufigen Kontakt zu einem weiten Kreis an Menschen hat und ausreichend interessiert daran ist, sich als öffentlicher Charakter zu etablieren“" (Jacobs 2013: 148, eigene Übersetzung).[9] Um solch ein „öffentlicher Charakter“ werden zu können, ist es vor allem wichtig, öffentlich zu sein; das heißt, präsent zu sein und viel mit verschiedenen Menschen zu sprechen (ebd.). Fréderic, ein weiterer Verkäufer, beschreibt, wie der Guthabenverkauf ihn und seinen Kollegen im Alltag beeinflusst:

„[Was sich durch die cabine verändert hat ist, dass] wir viele Menschen kennen, die nicht zu unserem sozialen Rang gehören oder die eigentlich nicht in unserem Milieu sind. Es gibt Leute, wenn wir Dir ihre Stellung verraten würden, oh la la! Aber eben jene Leute [...] sitzen mit uns auf denselben Bänken der cabine, unter demselben Schirm, und dann reden wir so miteinander, ohne Probleme. Oft sind es CEOs, Leute, die bei den Steuern arbeiten, Leute, die Berater des Ministers sind. Aber wenn sie hier an die cabine kommen, fühlen sie sich so wohl in der Kommunikation, wie wir reden, wie wir sie behandeln!“ (Interview mit Fréderic, Yopougon, 11. Oktober 2017)

Die Etablierung von Guthabenverkaufsstellen am Straßenrand, an lokalen Marktplätzen oder vor dem eigenen Haus ermöglicht es so, Räume der Geselligkeit zu schaffen, die soziales Kapital generieren (Vuarin 1994; Portes 1998).

Untermauert wird dies durch konkrete NachbarInnenschaftsdienste der Guthabenverkäufer: So bewahrte David etwa Schlüssel oder Geld für NachbarInnen auf, um sie später an Mitglieder ihrer Familien weiterzugeben. Die cabine war eine Anlaufstelle, um die herum sich Praktiken der „lokalen Hilfsbereitschaft“ (Kusenbach 2006: 291 ff., eigene Übersetzung) herauskristallisierten. Davon profitierte auch David, der sich durch diese Praktiken des neighboring, der NachbarInnenschaftlichkeit, als junger Mann ein positives Image und einen guten Ruf aufbauen konnte. Dieser wiederum wurde zu symbolischem Kapital[10] (Bourdieu 1977). Dank seines guten Rufes dachte 
man an David und berücksichtigte ihn, wenn sich eine Gelegenheit auftat oder es Ressourcen zu verteilen gab. So luden etwa ein paar NachbarInnen David und einen Kollegen zu einer warmen Mahlzeit ein oder man ließ David von Arbeitsgelegenheiten wissen.

Alle drei Aspekte zeigen, wie junge Erwachsene über den Verkauf von Guthaben ihre soziale Existenz in der Stadt weiterweben: Sie leisten erstens Statusarbeit und schaffen sich Zeugnisse ihres guten Rufes. Sie kreuzen zweitens verschiedene soziale Kreise und profitieren vom darin enthaltenen sozialen Kapital. Sie bleiben drittens Mitglieder von NachbarInnenschaftsnetzwerken, die es ihnen ermöglichen, über die Runden zu kommen. Zentral ist dabei ihre Etablierung als „öffentlicher Charakter“. Die Orte, an denen sie sitzen und verkaufen, begünstigen dies: an Straßenecken, auf Bürgersteigen, vor Hauseingängen.

\section{Wert abschöpfen: \\ Die Bedeutung der sozialen Infrastrukturen von Guthaben- verkäuferInnen für Telekommunikationsunternehmen}

Die besondere Stellung der cabines als Orte sozialen Austauschs und städtischer Öffentlichkeit ist funktional für zwei Seiten: für die GuthabenverkäuferInnen ebenso wie für die Telekommunikationsunternehmen, die daran interessiert sind, als Plattformen im alltäglichen Leben präsent zu sein. Das Verkaufen von Guthaben in sozialen Netzwerken, das als improvisierte Praxis begann, ist in den 2010er-Jahren Teil einer Marktstrategie der Telekommunikationsunternehmen geworden. Für Unternehmen wie Orange, Moov oder MTN sind die cabines eine Möglichkeit, ihre Produkte auf die Straße und in die Wohngebiete zu bringen. Sie nutzen die cabines als Plattformen für ihre Markenpolitik und als Vehikel zur Erschließung von neuem sozialem Terrain für den Verkauf ihrer Dienstleistungen und, um mehr KundInnen an ihre Kommunikationsplattformen zu binden. Zum Zeitpunkt der Forschung waren die GuthabenverkäuferInnen MikrounternehmerInnen ohne Arbeitsverträge mit den Telekommunikationsunternehmen. Sie registrierten sich lediglich beim jeweiligen Anbieter mit ihrem Personalausweis und erwarben eine professionelle SIM-Karte mit erweiterten Transaktionsmöglichkeiten. Dennoch waren GuthabenverkäuferInnen ein wichtiger Spielstein der Konzerne in der Erschließung von städtischen Räumen, wie die Gespräche mit leitenden Mitarbeitern in einem Subunternehmen eines Telekommunikationsunternehmens verdeutlichten. Durch die digitale Plattform-Technologie war es möglich, die Bewertungen und Verkaufszahlen jedeR einzelnen VerkäuferIn per SIM-Karte zu beobachten. Die Unternehmen konnten SIM-Karten aktivieren und deaktivieren und auf der Grundlage von Verkaufszahlen einzelne GuthabenverkäuferInnen belohnen. Besonders erfolgreiche VerkäuferInnen bekamen ,Geschenke‘ wie Sonnenschirme, Produktinformationstafeln oder T-Shirts mit dem Brand des Telefonanbieters. Trotz ihrer losen Kopplung an die Telefonanbieter waren die GuthabenverkäuferInnen als informelle MikrounternehmerInnen „zentrale Akteure“ beim Verkauf von Kommunikations- und Datenguthaben. So formulierte es im Interview der Leiter eines Subunternehmens, das ein Telekommunikationsunternehmen mit der Guthabenverteilung in einem 
Stadtteil von Abidjan beauftragt hatte (Interview mit CEO, Yopougon, 11. Oktober 2017). Über die GuthabenverkäuferInnen erreichten die Telefonanbieter Bevölkerungsgruppen, die sonst nur schwer mobilisierbar waren. Zudem ermöglichten sie ihnen das Branding im öffentlichen Raum. Die Marke des jeweiligen Telekommunikationsunternehmens war in dieser Hinsicht ein Teil der zu verteilenden Ware. Die GuthabenverkäuferInnen waren, wenn auch nicht offiziell angestellt, die sichtbaren RepräsentantInnen dieser Marke im Tagesgeschäft in Abidjan. Die Subunternehmen der Telekommunikationsunternehmen versuchten daher, die GuthabenverkäuferInnen mittels eines Codes of Conduct zu TrägerInnen des Markenimages zu machen: Sie wurden zu Aushängeschildern für eine Markenöffentlichkeitsarbeit, ohne dafür entlohnt zu werden. Während eines Treffen mit VertreterInnen eines dieser Subunternehmens wurden GuthabenverkäuferInnen dazu animiert, sich folgende Verhaltenskodizes (,Konventionen') zu Herzen zu nehmen: Präsenz, Positionierung, Förderung (promotion) und Sauberkeit (propreté) (Feldnotiz, Yopougon, 17. März 2017). Zudem waren die,Geschenke', die die VerkäuferInnen erhielten (also die Sonnenschirme, Produkttafeln, T-Shirts und Notizhefte), mit Markennamen und dem entsprechenden Corporate Design gespickt. Damit wurde die Grundausstattung der GuthabenverkäuferInnen zu einem Teil der Markenpolitik.

Die eben skizzierten Verkaufsstrategien der Telekommunikationsunternehmen und ihrer Subunternehmen zeigen, wie informelle Arbeit normalisiert (Meagher/Lindell 2013) und in formale Wertschöpfungsprozesse eingebunden wird. Diese Informalisierung hat vor allem zur Folge, dass ein großer Teil der Arbeitszeit nicht entlohnt wird, etwa das Warten auf Kundschaft, die zur KundInnenbindung notwendige Beziehungsarbeit und die implizite Marken-Werbung. Zudem werden Risiken outgesourct - also auf die VerkäuferInnen abgewälzt. Bei Krankheit, Diebstahl oder finanziellen Krisen übernehmen die Unternehmen offiziell keinerlei Verantwortung. Dabei zeichnet sich ab, wie die Rollen der VerkäuferInnen als KonsumentInnen und ProduzentInnnen miteinander verschwimmen (Ritzer/Jurgenson 2010): Die jungen Erwachsenen erhalten von den Telekommunikationsunternehmen Geschenke als Belohnung für gute Verkaufsleistungen, die dann zu einer Dienstleistung werden - der Sichtbarmachung der Marke. Ebenso deutlich wird die Regulierung und Disziplinierung von Arbeitskraft durch positive Anreize anstelle von Verträgen, die Rechte und Pflichten regeln. Die Marke wird dem Angebot eines guten Verkaufsservices vorangestellt. Das deutet an, wie sehr Telekommunikationsunternehmen darauf erpicht sind, die KundInnenbindung zu verbessern, indem sie auf Markentreue setzen. Ein beständiger und gut zugänglicher Service ist in diesem Zusammenhang Mittel zum Zweck. Allgemeiner formuliert, verschwimmen die Grenzen zwischen Produktions- und Reproduktionsräumen. Operationen, in denen Wert abgeschöpft wird, dringen tief in Alltagspraktiken ein (Gago/Mezzadra 2017; Knaus/Margies/Schilling i. E.). Das ermöglicht den Unternehmen eine Wertschöpfung, ohne dass sie die soziale Kooperation derer, die sie ausbeuten, direkt organisieren müssen (Gago/Mezzadra 2017: 579).

Kommunikationsinfrastrukturen und privatwirtschaftliche Ökonomien werden tagtäglich reproduziert, indem sie auf die Beziehungsdynamiken von GuthabenverkäuferInnen zurückgreifen. Diese können nur im 
Zusammenhang mit den spezifischen Moralökonomien rund um Arbeit und Status in Côte d'Ivoire verstanden werden. Gerade für die männliche urbane Mittelschicht ist formale Lohnarbeit ein wichtiges Statusmerkmal. Zugleich ist diese aber für viele junge Männer heutzutage praktisch unerreichbar. In den nachbarInnenschaftlichen und verwandtschaftlichen Versorgungsökonomien Abidjans ist es für diese jungen Erwachsenen daher besonders wichtig, ihren guten Ruf als strebsame, hilfsbereite und ehrwürdige Erwachsene zu behaupten. Die cabines sind für sie eine Möglichkeit, dieses symbolische und soziale Kapital zu generieren. Die von mir interviewten Guthabenverkäufer bleiben dabei in einem Übergang zwischen Jugend- und Erwachsenenalter, denn ihr Einkommen durch den Guthabenverkauf reicht nicht aus, um sich davon eine eigene Existenz aufzubauen oder gar eine Familie zu ernähren. Dieser Schwebezustand zwischen Jugend- und Erwachsenenalter ist für die Telekommunikationsunternehmen eine potenzielle Wertschöpfungsquelle: Sie docken an die informalisierten Guthabenverkaufsstellen an und integrieren sie in ihre Marketingpolitik. Dabei nutzen sie diese Knotenpunkte für ihre eigene Sichtbarkeitspolitik. Die Analyse zeigt Formen der Ausbeutung auf, die im digitalisierten Kapitalismus bedeutsam werden (Fourcade/Kluttz 2020): Unternehmen nutzen die Moralökonomien sozialer Beziehungen im städtischen Alltagsleben junger unterbeschäftigter Männer für ihre Zwecke. Sie werden damit zu Trittbrettfahrern der sozialen Infrastrukturen in Wohngebieten und an Straßenrändern.

\section{Fazit: TelefonguthabenverkäuferInnen als Teil digitaler Wertschöpfungsketten}

Die bisherige Analyse hat die Vielfalt von Formen menschlicher Arbeit gezeigt, mit der Kommunikationsinfrastrukturen aufgebaut und aufrechterhalten werden. Die Kommunikationsinfrastruktur lebt von der gegenseitigen Durchkreuzung verschiedener Märkte und Ökonomien - Handys und Guthaben zirkulieren als Geschenke, Waren und Kapital. Die Analyse ist auch auf die Sichtbarkeitspolitiken der interviewten Guthabenverkäufer und Telekommunikationsunternehmen eingegangen. Beide nutzen die cabines als Plattformen. Es wurde deutlich, wie die Telekommunikationsunternehmen in den Alltag der StadtbewohnerInnen hineinwirken. Dabei bleiben die BewohnerInnen ko-kreativ: Sie eignen sich digitale Technologien an und nutzen sie zur Überbrückung von Krisen der sozialen Reproduktion. Die Telekommunikationsunternehmen nutzen diese Praktiken wiederum für ihre Wertschöpfung. Sie nutzen Tätigkeiten, die von den Akteuren selbst nicht als Arbeit markiert werden. Den jungen Erwachsenen geht es vorrangig um Freundschaftsdienste, das Knüpfen von Bekanntschaften oder die Aufrechterhaltung ihres eigenen guten Rufes. Geld zu verdienen ist ihnen zwar auch wichtig, aber der Guthabenverkauf ist eher temporär und gelegentlich, um offen für neue Gelegenheiten zu sein, sobald sich eine „richtige Arbeit" findet.

Gig Work, Gelegenheitsjobs und Auftragsarbeit haben einelange Geschichte. In vielen Aspekten entspricht die über digitale Technologien vermittelte Gig Economy langfristigen Formen von Arbeit und Arbeitsmärkten in Afrika (Stevano 2020). Die nähere Beleuchtung der Guthabenökonomie in Abidjan 
ist daher fruchtbar für aktuelle Diskussionen über Digitalisierung, Arbeit und Stadt: Zum einen sensibilisiert sie dafür, die zunehmende Bedeutung von Plattformen und Internetkonzernen für längerfristige Infrastruktur-Politiken zu kontextualisieren. Es waren die Telekommunikationsunternehmen, die als Infrastrukturbetreiber den Weg für den Aufstieg der Internetkonzerne und der Plattformtechnologien ebneten (Schiller 1999; Staab 2019). Plattformen werden zunehmend zu wichtigen (städtischen) Infrastrukturen. Sie können in diesem Sinne aus der Perspektive der Infrastrukturforschung untersucht werden (Plantin et al. 2018; Richardson 2018). Dabei spielen die Vielfalt menschlicher Arbeit sowie die Verknüpfung und Zirkulation von Informationen eine zentrale Rolle (Leszczynski 2020).

Zum anderen verdeutlicht die Analyse die verschwimmenden Grenzen zwischen Konsumption und Produktion (Comaroff/Comaroff 2000; Ritzer 2015). Diese ist auch relevant etwa für Untersuchungen der Wertschöpfungsstrategien von Plattformen wie YouTube oder Snapchat (Hawker/Carah 2020; Postigo 2016). Die Auslagerung von Arbeitsmitteln und -risiken auf außerbetrieblich Wertschaffende - wie hier im Falle der GuthabenverkäuferInnen wirft die Frage nach der Verschärfung sozialer Ungleichheiten auf. Auch PlattformarbeiterInnen müssen ihre Arbeitsmittel und Arbeitsorte meist privat organisieren und instand halten. Wie sich dies auf Haushaltsökonomien und Beziehungskonstellationen in Familien und Freundschaften auswirkt, sollte weiter untersucht werden (Schilling 2020). Die Analyse zeigt die Bedeutung informeller Hierarchien und Autoritätsbeziehungen sowie die Konventionen und Rituale, die Gig-ArbeiterInnen aktivieren, um sich zu organisieren. Diese Dynamiken sollten auch in weiteren Forschungen zur Gig Economy untersucht werden. Insbesondere Formen der Reziprozität und des Gabentausches (Fourcade/Kluttz 2020) sowie die Verschränkung materieller Transaktionen mit Beziehungssemantiken von FreundInnenschaft oder Familie gewinnen in der Plattformökonomie neu an Bedeutung - genannt seien hier die Stichworte Sharing Economy oder Crowdsourcing (vgl. u. a. Ravenelle 2017).

Die Publikation dieses Beitrags wurde durch die Deutsche Forschungsgemeinschaft (DFG) und den Open-Access-Fonds der Humboldt-Universität zu Berlin ermöglicht.

\section{Endnoten}

[1] Die Studie ist Teil meines Promotionsprojektes, das die Deutsche Forschungsgemeinschaft (DFG) im Rahmen eines Stipendiums im Internationalen Graduiertenkolleg „The World in the City" am Center for Metropolitan Studies der Technischen Universität Berlin gefördert hat.

[2] Praktiken beschreiben in einem Kontext eingebettete, regelmäßige Handlungen, ,inkorporierte Wissensordnungen“ (Schäfer 2013: 21), das heißt „Know-how abhängige und von einem praktischen, Verstehen“ zusammengehaltene Verhaltensroutinen“ (Reckwitz 2003: 289, zitiert in Schäfer 2013: 22).

[3] Das neoklassische wirtschaftswissenschaftliche Paradigma des homo oeconomicus geht von Ressourcen maximierenden, rationalen Akteuren aus, die ihre Entscheidungen auf der Grundlage stabiler Präferenzen treffen (vgl. Rogall 2006).

[4] Orange Bank. Nos comptes bancaires, https://www.orangebank.fr (letzter Zugriff am 1.5.2020). 
[5] Das Orange Fab Côte d'Ivoire ist ein Programm und eine Plattform, die jungen StartUps Infrastruktur, Finanzierung und Netzwerke anbietet, um deren Geschäftsidee weiterzuentwickeln, http://www.orangefab.ci (letzter Zugriff am 1.5.2020).

[6] http://www.gouv.ci/doc/presse/1560502545COLLECTE-DES-COTISAIONS-DESASSURES-POUR-LE-COMPTE-DE-COUVERTURE-MALADIE-UNIVERSELLE.pdf (letzter Zugriff am 1.5.2020).

[7] https://www.facebook.com/jumia.ci/posts/faites-le-plein-de-bonus-en-commandantavec-orange-money-sur-jumia-100o-fcfa-de-/963181047148474/ (letzter Zugriff am 1.5.2020).

[8] Anmerkung: Dabei sind sie nicht die einzige Gruppe an StadtbewohnerInnen, die Guthaben verkaufen.

[9] Ich danke an dieser Stelle Rika Febriyani, die mich bei der gemeinsamen Sichtung meines Fotomaterials zu den Guthabenverkäufern auf diese Referenz aufmerksam gemacht hat.

[10] Nach Bourdieu ist symbolischen Kapital (d. h. Prestige, Ansehen), „Kredit im weitesten Sinne des Wortes [...], d.h. eine Art Vorschuss, den die Gruppe allein denjenigen gewähren kann, die ihr die besten materiellen und symbolischen Garantien geben“ (Bourdieu 1977: 181). Es entsteht in Beziehungen: Gewöhnliche Eigenschaften von Akteuren (z. B. physische Kraft, Reichtum usw.) verwandeln sich in symbolisches Kapital der Anerkennung und werden symbolisch effizient, weil sie als solche erkannt werden. Das symbolische Kapital erscheint ,in den sozialen Beziehungen zwischen Eigenschaften, die ein Akteur besitzt, und anderen Akteuren, die mit angemessenen Wahrnehmungskategorien ausgestattet sind“ (Bourdieu 1998: 104).

\section{Autor_innen}

Hannah Schilling ist Stadtsoziologin und arbeitet zu städtischen Ökonomien und sozialer Ungleichheit in globaler Perspektive. Sie promovierte mit einer vergleichenden Analyse von Prekarisierungsprozessen unter jungen Erwachsenen in digitaler Arbeit in Abidjan und Berlin. schillha@hu-berlin.de

\section{Literatur}

Altenried, Moritz (2017): Die Plattform als Fabrik. Crowdwork, Digitaler Taylorismus und die Vervielfältigung der Arbeit. In: PROKLA. Zeitschrift für kritische Sozialwissenschaft 47/187, 175-192.

Angelo, Hillary / Hentschel, Christine (2015): Interactions with infrastructure as windows into social worlds. A method for critical urban studies: Introduction. In: City 19/2-3, 306-312.

Appadurai, Arjun (2014): Foreword. In: Stephen Graham / Colin McFarlane (Hg.), Infrastructural lives. Urban infrastructure in context. Abingdon/New York: Routledge.

Autorité de Régulation des Télécommunications/TIC de Côte d'Ivoire (ARTCI) (2015): Données statistiques du secteur des télécommunications en Côte d'Ivoire. Troisième trimestre 2015. https://www.artci.ci/images/stories/pdf/rapport_activite/statistiques_ annuelles_2015.pdf, (letzter Zugriff am 2.3.2021).

Bazin, Laurent / Gnabéli, Roch Yao (1997): Le travail salarié, un modèle de décomposition? In: Bernard Contamin / Harris Memel-Fote (Hg.), Le modèle ivoirien en questions. Crises, ajustements, recompositions. Paris: Karthala, 689-705.

Bennett, Jane (2005): The agency of assemblages and the North American blackout. In: Public Culture 17/3, 445-465.

Berlant, Lauren (2016): The commons. Infrastructures for troubling times. In: Environment and Planning D: Society and Space 34/3, 393-419.

Bourdieu, Pierre (1977): Structures, habitus, power. Basis for a theory of symbolic power. Outline of a theory of practice. Cambridge/New York: Cambridge University Press, 159-197.

Bourdieu, Pierre (1998): The economy of symbolic goods. Practical reason. On the theory of action. Stanford: Stanford University Press, 92-124. 
Bourdieu, Pierre (2000): Making the economic habitus. Algerian workers revisited. In: Ethnography 1/1, 1466-1381.

Charmaz, Kathleen C. (2006): Constructing grounded theory: A practical guide through qualitative analysis. London/Thousand Oaks: Sage.

Comaroff, Jean / Comaroff, John (2000): Millennial capitalism. First thoughts on a second coming. In: Public Culture 12/2, 291-343.

Cutolo, Armando (2015): Giovani, cadetti et vieux pères. Guerra e riproduzione sociale in Costa d'Avorio. In: Anthropologia II/1, 61-84.

Dolata, Ulrich (2015): Volatile Monopole. Konzentration, Konkurrenz und Innovationsstrategien der Internetkonzerne. In: Berliner Journal für Soziologie 24/4, 505-529.

Eisenstadt, Shmuel N. / Roniger, Louis (1980): Patron-Client relations as a model of structuring social exchange. In: Comparative Studies in Society and History 22/01, 42.

Eyert, Florian / Irgmaier, Florian / Ulbricht, Lena (2020): Extending the framework of algorithmic regulation. The Uber case. In: Regulation \& Governance, https://doi. org/10.1111/rego.12371.

Fancello, Sandra / Mary, André (2018): Institutions du pardon et politiques de la délivrance en Afrique de l'Ouest. In: Journal des Africanistes 88/2, 76-99.

Ferguson, James / Li, Tania Murray (2018): Beyond the „proper job“. Political-economic analysis after the century of labouring man. Working Paper 51. PLAAS, UWC (Institute for Poverty, Land and Agrarian Studies, University of Western Cape): Cape Town. 1-22.

Fourcade, Marion / Kluttz, Daniel N. (2020): A Maussian bargain. Accumulation by gift in the digital economy. In: Big Data \& Society 7/1, 1-16.

Gago, Verónica / Mezzadra, Sandro (2017): A critique of the extractive operations of capital. Toward an expanded concept of extractivism. In: Rethinking Marxism 29/4, 574-591.

Gibson-Graham, Julie Katherine (2008): Diverse economies. Performative practices for „other worlds“. In: Progress in Human Geography 32/5, 613-632.

Graham, Stephen (2009): Disrupted cities. When infrastructure Fails. New York: Routledge.

Graham, Steve / Marvin, Simon (2001): Splintering urbanism. Networked infrastructures, technological mobilities and the urban condition. London/New York: Routledge.

Griesbach, Kathleen / Reich, Adam / Elliott-Negri, Luke / Milkman, Ruth (2019): Algorithmic control in platform food delivery work. In: Socius $5,1-5$.

Gudeman, Stephen (2008): Economy's tension. The dialectics of market and economy. New York/Oxford: Berghahn Books.

Guérin, Isabelle (2014): Juggling with debt, social ties, and values: The everyday use of microcredit in rural south India. In: Current Anthropology 55/S9, S40-S5O.

Hamanys Broux de Ismael, Koffi (2016): La téléphonie mobile à Abidjan. Usagers, usages et représentations. Saarbrücken: Éditions Universitaires Européennes.

Hann, Chris (2018): Economic anthropology. In: Hilary Callan (Hg.), The international encyclopedia of anthropology. Oxford: Wiley, 1-16.

Hart, Keith (1973): Informal income opportunities and urban employment in Ghana. In: The Journal of Modern African Studies 11/1, 61-89.

Hawker, Kiah / Carah, Nicholas (2020): Snapchat's augmented reality brand culture. Sponsored filters and lenses as digital piecework. In: Continuum, https://doi.org/10.108 o/10304312.2020.1827370.

Jacobs, Jane (2013): The uses of sidewalks. Contact. In: Michael Larice / Elizabeth Macdonald (Hg.), The urban design reader. Abingdon: Routledge, 139-151.

Kamga, Osée (2012): Digital technologies and imaginary of uses in the developing world. Mobile phone inroads in the Côte d'Ivoire's social fabric. In: Vikas Kumar / Jakob Svensson (Hg.), Proceedings of conference on Mobile Communication for Development (M4D) 2012 in New Delhi, India, 28-29 February 2012. Karlstad: Karlstad University Studies, 377-381.

Kendall, Jake / Maurer, Bill / Machoka, Phillip / Veniard, Clara (2012): An emerging platform. From money transfer system to mobile money ecosystem. In: innovaTions 6/4, 49-64.

Kirchner, Stefan (2019): Arbeiten in der Plattformökonomie. Grundlagen und Grenzen von „Cloudwork“ und „Gigwork“. In: Kölner Zeitschrift für Soziologie und Sozialpsychologie $71 / 1,3-25$.

Kiyindou, Alain / Anaté, Kouméalo / Capo Chichi, Alain (Hg.) (2015): Quand l'Afrique réinvente la téléphonie mobile. Collection „Études africaines“. Paris: L’Harmattan. 
Knaus, Katharina / Margies, Nina / Schilling, Hannah (i. E.): Thinking the city through work. Blurring boundaries of production and reproduction in the age of digital capitalism. In: CITY.

Kouakou, Clément Kouadio (2011): Urban youth labour. Supply and employment policy in Côte d'Ivoire. African Economic Research Consortium Research Paper 240. Nairobi: African Economic Research Consortium.

Kouakou, Clément Kouadio / Koba, Adébo (2015): L’emplois des jeunes en Côte d’Ivoire. Une étude diagnostique. Ottawa: Centre de Recherches pour le Développement International.

Kusenbach, Margarethe (2006): Patterns of neighboring. Practicing community in the parochial realm. In: Symbolic Interaction 29/3, 279-306.

Laffont, Jean-Jacques / N'Guessan, Tchétché (2002): Telecommunications reform in Côte d'Ivoire. Policy Research Working Papers. o. O.: The World Bank.

Larkin, Brian (2013): The politics and poetics of infrastructure. In: Annual Review of Anthropology 42, 327-343.

Leszczynski, Agnieszka (2020): Glitchy vignettes of platform urbanism. In: Environment and Planning D: Society and Space 38/2, 189-208.

Lourenço-Lindell, Ilda (2001): Social networks and urban vulnerability to hunger. In: Arne Tostensen / Inge Tvedten / Mariken Vaa (Hg.), Associational life in African cities. Popular responses to the urban crisis. Uppsala: Nordiska Afrikainstitutet, 30-45.

Mains, Daniel (2013): Friends and money. Balancing affection and reciprocity among young men in urban Ethiopia. In: American Ethnologist 40/2, 335-346.

Manou-Savina, Annie / Antoine, Philippe / Dubresson, Alain / Yapi Diahou, Alphonse (1985): Les en-haut des en-bas et les en-bas des en-haut. Classes moyennes et urbanisation à Abidjan (Côte d'Ivoire). In: Revue Tiers Monde 26/101, 55-68.

Marcelli, Enrico / Williams, Colin / Joassart, Pascale (Hg.) (2010): Informal work in developed nations. New York: Routledge.

Marie, Alain (1997): Individualisation. Entre communauté et société l'avènement du sujet. In: Alain Marie / Robert Vuarin (Hg.), L’Afrique des individus. Itinéraires citadins dans l'Afrique contemporain. Paris: Karthala, 407-436.

Matlon, Jordanna (2014): Côte d'Ivoire. The symbolic capital of the mobile phone. In: Global Dialogue 4, 33-34.

Matlon, Jordanna (2016): Racial capitalism and the crisis of black masculinity. In: American Sociological Review 81/5, 1014-1038.

Mbembe, Achille / Roitman, Janet (1995): Figures of the subject in times of crisis. In: Public Culture 7/2, 323-352.

Meagher, Kate / Lindell, Ilda (2013): ASR forum. Engaging with African informal economies. Social inclusion or adverse incorporation? In: African Studies Review 56/03, 57-76.

Mezzadra, Sandro / Neilson, Brett / Cukier, Alexis (2018): Entre extraction et exploitation. Des mutations en cours dans l'organisation de la coopération sociale. In: Actuel Marx 63/1, 97 .

Muniesa, Fabian / Millo, Yuval / Callon, Michel (2007): An introduction to market devices. In: The Sociological Review 55/2 (Beilage), 1-12.

Nassa Dabié, Désiré Axel (2012): Contribution de la téléphonie mobile à la dynamisation du commerce informel dans la commune d'Adjamé à Abidjan en Côte d'Ivoire. In: Bulletin de l'Association de Géographes Français 89/4, 597-613.

Neves, David / Toit, Andries du (2012): Money and sociality in South Africa's informal economy. In: Africa 82/1, 131-149.

Plantin, Jean-Christophe / Lagoze, Carl / Edwards, Paul / Sandvig, Christian (2018): Infrastructure studies meet platform studies in the age of Google and Facebook. In: New Media \& Society 20/1, 293-310.

Portes, Alejandro (1998): Social capital. Its origins and applications in modern sociology. In: Annual Review of Sociology 24/1, 1-24.

Postigo, Hector (2016): The socio-technical architecture of digital labor. Converting play into YouTube money. In: New Media \& Society 18/2, 332-349.

Przyborski, Aglaja / Wohlrab-Sahr, Monika (2009): Qualitative Sozialforschung. Ein Arbeitsbuch. München: Oldenbourg.

Ravenelle, Alexandrea J. (2017): Sharing economy workers. Selling, not sharing. In: Cambridge Journal of Regions, Economy and Society 10/2, 281-295.

Reckwitz, Andreas (2002): Toward a theory of social practices. A development in culturalist theorizing. In: European Journal of Social Theory 5/2, 243-263. 
Reckwitz, Andreas (2003): Grundelemente einer Theorie sozialer Praktiken. Eine sozialtheoretische Perspektive. In: Zeitschrift für Soziologie 32/4, 282-301.

Rekhviashvili, Lela (2015): Counterbalancing marketization informally. Institutional reforms and informal economic practices in Georgia (2003-2012). Dissertation. Budapest: Central European University.

Richardson, Lizzie (2017): Sharing as a postwork style. Digital work and the co-working office. In: Cambridge Journal of Regions, Economy and Society 10/2, 297-310.

Richardson, Lizzie (2018): Platforms as urban technology? In: Mediapolis. A Journal of Cities and Culture 3/4. https://www.mediapolisjournal.com/2018/11/platforms-asurban-technology (letzter Zugriff am 22.4.2020).

Ritzer, George (2015): Prosumer capitalism. In: The Sociological Quarterly 56/3, 413-445.

Ritzer, George / Jurgenson, Nathan (2010): Production, consumption, prosumption: The nature of capitalism in the age of the digital „prosumer“. In: Journal of Consumer Culture 10/1, 13-36.

Robinson, Jennifer (2002): Global and world cities. A view from off the map. In: International Journal of Urban and Regional Research 26/3, 531-554.

Robinson, Jennifer (2015): Thinking cities through elsewhere. Comparative tactics for a more global urban studies. In: Progress in Human Geography 40/1, 1-27.

Rogall, Holger (Hg.) (2006): Neoklassik. Volkswirtschaftslehre für Sozialwissenschaftler. Eine Einführung. Wiesbaden: VS Verlag für Sozialwissenschaften, 57-68.

Rossi, Ugo (2019): The common-seekers. Capturing and reclaiming value in the platform metropolis. In: Environment and Planning C: Politics and Space 37/8, 1418-1433.

Roy, Ananya / AlSayyad, Nezar (Hg.) (2004): Urban informality. Transnational perspectives from the Middle East, Latin America, and South Asia. Lanham/Berkeley: Lexington Books.

Schäfer, Hilmar (2013): Die Instabilität der Praxis. Reproduktion und Transformation des Sozialen in der Praxistheorie. Weilerswist: Velbrück Wissenschaft.

Schilling, Hannah (2020): Aller se chercher. Urban youth's making of livelihood in unstable work in Abidjan and Berlin. Berlin: Humboldt-Universität zu Berlin.

Schilling, Hannah / Dembélé, Ousmane (2019): Jeunes entreprenants de la téléphonie mobile à Abidjan. Du lien social entrepreneurial au service de l'exploitation économique. In: Politique Africaine 153/1, 181-201.

Schiller, Dan (1999): Digital capitalism networking the global market system. Cambridge: MIT Press.

Schiller, Dan (2014): Digital depression. Information technology and economic crisis. Urbana/Chicago: University of Illinois Press.

Schmidt, Florian A. (2016): Arbeitsmärkte in der Plattformökonomie. Zur Funktionsweise und den Herausforderungen von Crowdwork und Gigwork. Bonn: Friedrich-Ebert-Stiftung.

Simone, AbdouMaliq (2004): People as Infrastructure. Intersecting Fragments in Johannesburg. In: Public Culture 16/3, 407-429.

Simone, AbdouMaliq (2010): City Life from Jakarta to Dakar. Movements at the Crossroads. New York: Routledge.

Slater, Don / Tonkiss, Fran (2001): Market society. Markets and modern social theory. Cambridge: Polity Press.

Smith, Adrian / Stenning, Alison (2006): Beyond household economies. Articulations and spaces of economic practice in postsocialism. In: Progress in Human Geography 30/2, 190-213.

Staab, Philipp (2019): Digitaler Kapitalismus. Markt und Herrschaft in der Ökonomie der Unknappheit. Berlin: Suhrkamp.

Staab, Philipp / Nachtwey, Oliver (2016): Market and labour control in digital capitalism. In: tripleC: Communication, Capitalism \& Critique. Open Access Journal for a Global Sustainable Information Society 14/2, 457-474.

Star, Susan Leigh (1999): The ethnography of infrastructure. In: American Behavioral Scientist 43/3, 377-391.

Stefano, Valerio de (2015): The rise of the ,just-in-time workforce“. On-demand work, crowd work and labour protection in the „gig-economy“. Rochester: Social Science Research Network.

Stevano, Sara (2020): „Gig economies“ in Africa. Continuity or change? In: Futures of Work 12. https://futuresofwork.co.uk/2020/04/o1/gig-economies-in-africa-continuityor-change (letzter Zugriff am 30.4.2020). 
Thieme, Tatiana (2018): The hustle economy. Informality, uncertainty and the geographies of getting by. In: Progress in Human Geography 42/4, 529-548.

Tonkiss, Fran (2015): Afterword. Economies of infrastructure. In: City 19/2-3, 384-391.

Touré, Mamoutou (2010): Logique des contrats et réalité du marché. La filière de la grande distribution des cartes de recharge téléphoniques. In: Les Cahiers d'Outre-Mer. Revue de géographie de Bordeaux 63/251, 403-418.

Tranber Hansen, Karen / Vaa, Mariken (Hg.) (2004): Reconsidering informality. Perspectives from urban Africa. Stockholm: Nordic Africa Institute.

Tsing, Anna (2013): Sorting out commodities. How capitalist value is made through gifts. In: HAU Journal of Ethnographic Theory 3/1, 21-43.

Viti, Fabio (2013): Travail et apprentissage en Afrique de l'Ouest. Sénégal, Côte d'Ivoire, Togo. Paris: Karthala.

Vuarin, Robert (1994): L'argent et l'entregent. In: Cahier des Sciences Humaines 30/1-2, 255-273.

Wood, Alex J. / Graham, Mark / Lehdonvirta, Vili / Hjorth, Isis (2019): Good gig, bad gig. Autonomy and algorithmic control in the global gig economy. In: Work, Employment and Society 33/1, 56-75.

Zelizer, Viviana A. (1996): Payments and social ties. In: Sociological Forum 11/3, 481-495.

\section{Interviewquellen}

Interview mit Boris, Yopougon, 8. März 2016

Interview mit CEO, Yopougon, 11. Oktober 2017

Interview mit David, Koumassi, 13. Oktober 2017

Interview mit François, Yopougon, 19. Februar 2016

Interview mit Fréderic, Yopougon, 11. Oktober 2017

Interview mit Séverin, Koumassi, 3. März 2016

\section{Young urbanites between improvisation and exploitation. Abidjan's call box workers as nodal points in extractive opera- tions of digital capitalism}

This article takes the mobile phone economy in Abidjan, Côte d'Ivoire as a starting point to problematize urban value creation processes in digital capitalism. To what extent do credit vendors become nodes of urban infrastructures? Can forms of exploitation also be identified in the appropriation practices of new technologies? The article links the debate on platform urbanism with studies on urban infrastructures and is based on a case study on the precarisation of young adults in Abidjan's credit economy between 2015 and 2017, using empirical evidence from indepth interviews with about 30 men between 18 and 35 years. In the analysis, I show the entanglement of forms of circulation in practices of selling credit and the functionality of cabines as platforms for visibility policies. Airtime sellers can establish their reputation as „public characters“, but the call boxes also serve as platforms for visibility policies and marketing. Finally, I draw attention to closure processes that are emerging in the course of the current platformisation of the communication infrastructure. In the past, companies have used the improvisation and appropriation of digital technologies by credit vendors as nodes in urban neighborhoods to expand markets. Today, the daily work of the credit sellers threatens to become superfluous, which makes the question of social reproduction in platform urbanism even more acute. 